\title{
Contorting a heavy and naturally curved elastic rod
}

\begin{abstract}
Arnaud Lazarus, ${ }^{a}$ Jay T. Miller, ${ }^{b}$ Matthew M. Metlitz ${ }^{a}$ and Pedro M. Reis*ab
We investigate how the configurations obtained from the writhing of a heavy elastic rod are influenced by its intrinsic natural curvature. To this end, we perform a combination of numerics and precision model experiments on the compression or twisting of a thin rod. The 'softness' of these single elastic filaments stems from their slenderness (high aspect ratio), which allow for geometrically nonlinear compliant modes that can accommodate large deformations. We uncover the original mechanism that the presence of a body force (gravity in our case) delays the effect of natural curvature, which results from the complex interplay between geometrical constraints, elasticity and weight. We rationalize our experimental results by coupling the predictive power of a numerical method of our own, with classic theory for elastic rods under large deformations. This preponderance of geometry is relevant in systems over a wide range of length scales where curvature and body-forces often co-exist; from engineered rod-like structures such as wires, cables, and pipelines, to natural macromolecules, flagella, fibers and tendrils.
\end{abstract}

\section{Introduction}

Predictively understanding the mechanics of soft structures comprising of slender elastic rods that can deform into loops and tangles, as well as rationalizing their mechanical response, is important in many practical problems that span a wide range of length scales. Examples include, to mention just a few: the complex physical behavior of DNA strands ${ }^{1-3}$ and DNA rings,${ }^{4-6}$ the inversion of chirality in helical bacterial flagella, ${ }^{7}$ morphogenesis of tendril perversion in climbing plants ${ }^{\mathbf{8 - 1 0}}$ and damage due to localized deformation in twisted marine cables. ${ }^{\mathbf{1 1}, \mathbf{1 2}} \mathrm{A}$ commonality across these various systems is that they can exhibit geometrically nonlinear behavior. Moreover, rod-like structures can possess an intrinsic curvature (i.e. they are not naturally straight) and their elasticity may couple with body forces such as hydrodynamic loading, electro-magnetic interactions (at small scales), or gravity (at macroscopic scales). These individual filamentary structures may also be assembled into larger networks to produce more complex soft materials and structures.

The classic problem of writhing of a thin $\operatorname{rod}^{13,14}$ (quasistatically varying the end-to-end displacement or rotation of its extremities, see Fig. 1) has become a canonical model system in which to investigate the equilibrium configurations, stability and spatial localization of contorted filamentary structures. ${ }^{\mathbf{1 5 - 1 8}}$ With particular focus during the 1990's, a large body of work has addressed the Kirchhoff's analogy, whereby the nonlinear differential equations for 3D elastica have a one-to-one map to

${ }^{a}$ Department of Mechanical Engineering, Massachusetts Institute of Technology, Cambridge 02139, USA

${ }^{b}$ Department of Civil and Environmental Engineering, Massachusetts Institute of Technology, Cambridge 02139, USA.E-mail: preis@mit.edu those of a spinning top. ${ }^{19}$ This interpretation has allowed for the theoretical framework and computational techniques from the field of dynamical systems $\mathbf{s}^{\mathbf{2 0 - 2 2}}$ to be ported to the study of the mechanical instabilities of thin rods. For example, buckling of a slender elastic filament under torsion can be attributed to a Hopf bifurcation, ${ }^{\mathbf{1 6}}$ and the rod's subsequent post-buckling configurations can be computed by calculating the associated bifurcation diagram.

Complex equilibrium states and their transitions during the writhing of naturally straight elastic rods undergoing large deflections have been explained statically and dynamically using the framework of Kirchhoff's equations for elastic rods. ${ }^{\mathbf{1 6 - 2 6}}$ Similarly, several studies rationalized the effect of intrinsic natural curvature on the inversion of helix handedness $^{\mathbf{9 1 0 , 2 7}}$ or the presence of one-twist-per-wave configurations ${ }^{28}$ in infinitely long twisted circular rods. Recently, more sophisticated numerical models have also considered phenomena

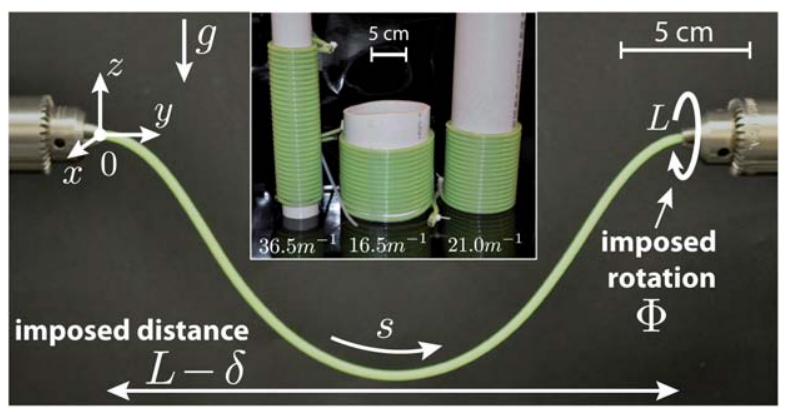

Fig. 1 The writhing experiment. A naturally curved elastomeric rod is clamped to two concentrically aligned drill chucks, which allow for the end-to-end displacement or rotation of the extremities to be imposed. Inset: fabrication process of the rods with custom natural curvature, $\kappa_{0}$. 
with self-contact, in this class of problems. ${ }^{10,12,26,29}$ Investigations in this realm, however, tend to be highly theoretical and experiments are primarily used to qualitatively validate the demonstrated results, ${ }^{16,25,28}$ rather than as a tool for discovery and precision quantification..$^{30-32}$ Inherent to these theoretical approaches is a need for simplifying assumptions (e.g. weightless, infinite length and perfectly straight rods), to ensure tractability of what, regardless, are already intricate systems involving a high level of technicality.

Here, we perform precision model experiments, coupled with our own numerical simulations, to investigate the original scenario when both a conservative body force (the rod's selfweight due to gravity, in our case) and intrinsic natural curvature are important ingredients. Our goal is to develop physical insight and gain a better understanding of practical and natural systems where the body force cannot be neglected, e.g. gravity in wires, cables, tubes and pipes. Moreover, these objects typically need to be spooled for storage and transport, which may irreversibly impart a natural curvature that must be treated as an independent variable. ${ }^{33-35}$

In our precision model experiments, we custom fabricate elastomeric rods with a set intrinsic natural curvature, $\kappa_{0}$, that is taken as one of the control parameters. The rod sample is then suspended between two horizontal concentric drill chucks, as illustrated in Fig. 1, and two sets of complementary experiments are performed. For different values of $\kappa_{0}$, the mechanical response of the thin rod is quantified by quasi-statically varying either (i) the end-to-end displacement (without rotation) or (ii) the end-to-end rotation (fixing the distance between clamps).

In parallel to the experiments, we also perform simulations (of the same scenario explored experimentally) using our own asymptotic numerical continuation method..$^{36}$ This method combines a geometrically-exact rod model with a semi-analytical perturbation technique to perform a continuation of the equilibrium branches and calculate the underlying bifurcations. After a direct comparison against experimental results, we can then take advantage of the predictive power of our simulations to explore quantities that cannot be readily accessed experimentally, such as the role of internal axial forces on the pattern formation process of the rod.

In both cases of our study (imposed displacement or rotation), we find that natural curvature does indeed qualitatively and quantitatively change the nature of the bifurcations and, consequently, affects the resulting $3 \mathrm{D}$ configurations of the deformed rod. Although a number of aspects of this system have been addressed in previous studies,,$^{5,9,10,28}$ we uncover the original effect that weight delays the effect of natural curvature. Below a critical value $\kappa_{0}^{\text {crit }}$, gravity balances the imposed geometry and the heavy rod can be considered as being naturally straight, albeit with a small imperfection $\kappa_{0}$. In contrast, above $\kappa_{0}^{\text {crit }}$, the effect of natural curvature is significant and sufficient to break the symmetries of the rod's pattern formation.

Our paper is organized as follows. We start by presenting the experimental apparatus and the computational approach that we use to investigate both versions of the writhing experiments. We then first focus on the case of imposed end-to-end displacement and rationalize the underlying physical ingredients of the writhing problem: elasticity, curvature and internal forces due to weight. To attest to the strength of the revealed mechanism, we then explore the second case of imposed end-to-end rotation through which we uncover similar trends. We finish by discussing and contrasting both scenarios.

\section{Experimental and numerical setup}

We fabricate our rods through casting, by injecting vinylpolysiloxane (VPS), an elastomer, into a flexible PVC tube of inner and outer diameters $D_{\mathrm{I}}=3.1 \mathrm{~mm}$ and $D_{\mathrm{O}}=5 \mathrm{~mm}$, respectively. The PVC mold tube is first wound around a cylinder of external radius $R_{\mathrm{e}}$ and then injected with VPS, which eventually crosslinks at room temperature [see inset of Fig. 1]. After a setting period of 24 hours, to ensure complete curing of the polymer, the outer flexible PVC pipe is cut to release the inner slender VPS elastic rod with a constant natural curvature $\kappa_{0}=1 /\left(R_{\mathrm{e}}+D_{\mathrm{O}} / 2\right)$, in the range $0<\kappa_{0}<60 \mathrm{~m}^{-1}$, and a circular cross-section $R=D_{\mathrm{I}} / 2=$ $1.55 \pm 0.22 \mathrm{~mm}$. We measure the Young's modulus of the elastomer to be $E=1.30 \pm 0.06 \mathrm{MPa}$, a volumic mass $\rho=1200 \pm$ $16 \mathrm{~kg} \mathrm{~m}^{-3}$ and a Poisson ratio of $\nu \approx 0.5$.

In our experiments, each naturally curved cast $\operatorname{rod}(L=30 \mathrm{~cm}$ long) is attached between two horizontal concentric drill chucks. We ensure that the sign of the constant intrinsic curvature, $\kappa_{0}$, is such that the rod naturally bends downwards, in the direction of gravity, $\boldsymbol{g}$. A photograph of the side view of the experimental setup is presented in Fig. 1. The boundary conditions of the rod are set to be rigidly clamped at both ends. The clamp located at the origin (curvilinear coordinate $s=0$ ) is completely fixed but the other clamp (at $s=L$ ) has two degrees of freedom: it can be rotated of an angle $\Phi$ or moved with a displacement $\delta$ towards the fixed left extremity [see Fig. 1]. Our two distinct experimental protocols consist of quasi-statically varying the displacement, $\delta$, (for an end-to-end rotation angle, $\Phi=0^{\circ}$ ) or increasing $\Phi$ (for $L-\delta=22 \mathrm{~cm}$ ). The resulting configurations of the rod are quantified through digital imaging by a camera located above the apparatus (top view). Representative configurations of these experiments at different values of the control parameters are shown in Fig. 2.

Our simulations are performed using an original asymptotic numerical continuation method to compute bifurcations of equilibria and stability of slender elastic rods. ${ }^{36}$ In this model, the $3 \mathrm{D}$ kinematics of the rod is treated in a geometrically exact way and we take into account deformations due to bending and twisting but assume no stretching (inextensibility condition). The boundary conditions are ensured to be perfectly clamped at both ends. For the material properties, we use an isotropic linear elastic model with the material properties measured independently in the experiments. The partial differential equations modeling the rod's equilibria are approximated with finite differences by discretizing the rod in 200 elements (a mesh sensitivity study was carried out to ensure that the results are unaffected by the element size). The subsequent set of algebraic equilibrium equations are solved with an asymptotic numerical method that gives interactive access to semi-analytical equilibrium branches, including state configurations, as shown in Fig. 2. In parallel, the local stability of equilibria is 


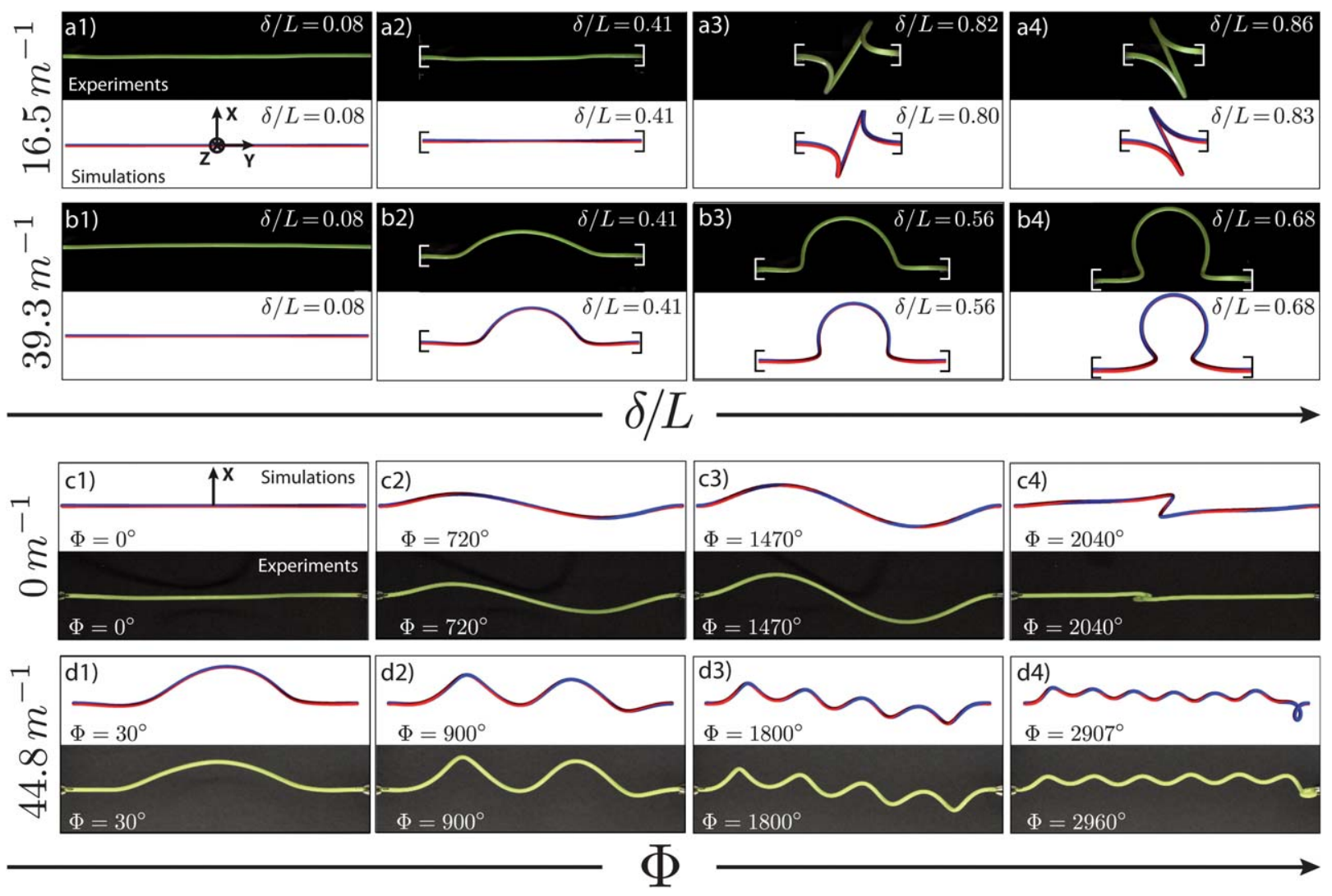

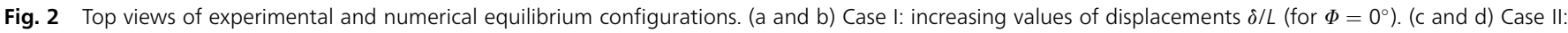

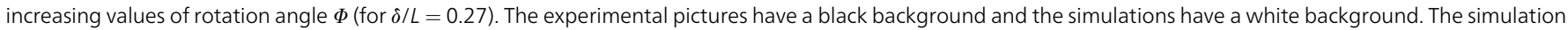

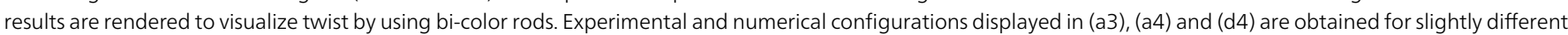
control parameters since the studied bifurcations are highly sensitive to imperfections. (a) $\kappa_{0}=16.5 \mathrm{~m}^{-1}$. (b) $\kappa_{0}=39.3 \mathrm{~m}^{-1}$. (c) $\kappa_{0}=0 \mathrm{~m}^{-1}$. (d) $\kappa_{0}=44.8 \mathrm{~m}^{-1}$.

assessed by computing the decay rate, $\sigma$, of the associated perturbation. The equilibrium is locally stable or unstable according to a positive or negative decay rate, respectively. This continuation method is implemented in the interactive pathfollowing and bifurcation analysis MATLAB package, ManLab. We have previously reported a detailed account of our numerical method elsewhere. ${ }^{36,37}$

\section{Writhing case I - imposed displacement}

We start by quasi-statically increasing the dimensionless displacement, $\delta / L$, while fixing the end-to-end rotation at $\Phi=$ $0^{\circ}$, for rods with different natural curvature, $\kappa_{0}$. Each experiment is initiated with the clamps separated by $L=30 \mathrm{~cm}$ (i.e. $\delta / L=0$ ) and terminated as soon as self-contact occurs (beyond which our numerics fail).

In Fig. 2a and $b$, we present a sequence of representative snapshots of the rod at different stages of displacement, for the experiments and simulations, with excellent agreement found between the two. Upon increasing $\delta / L$, the rod eventually bifurcates into an out-of-plane equilibrium configuration that is qualitatively different for different values of $\kappa_{0}$ (in Fig. 2a and b, two representative values of $\kappa_{0}=16.5 \mathrm{~m}^{-1}$ and $\kappa_{0}=39.3 \mathrm{~m}^{-1}$ are shown). For low curvature values, e.g. $\kappa_{0}=16.5 \mathrm{~m}^{-1}$, the clamped rod has an inflectional planar equilibrium shape in the $(y, z)$-plane, mostly due to its own weight, for a large range of displacements, $\delta / L$ [Fig. 2a1 and a2]. At large $\delta / L$, when both clamps approach each other, the rod buckles out of the plane into an equilibrium state that is anti-symmetric with respect to the clamp's centerline [Fig. 2a3 and a4], similarly to the secondary buckling of a weightless clamped rod, previously studied by others. ${ }^{\mathbf{2 4 , 2 6 , 3 2}}$ For a high values of $\kappa_{0}=39.3 \mathrm{~m}^{-1}$ and at small values of $\delta / L$ when the clamps are near their maximum separation distance, the equilibrium configuration is analogous to the planar shape observed for $\kappa_{0}=16.5 \mathrm{~m}^{-1}$ [Fig. 2b1]. When increasing $\delta / L$, however, the rod evolves into an out-of-plane equilibrium shape, shown in Fig. 2b2-b4, described by two helices with opposite handedness connected by a transition region where chirality is inverted, also known as perversion. ${ }^{8-10}$ In a case of a strong imposed geometry, the natural imperfection $\kappa_{0}=39.3 \mathrm{~m}^{-1}$ breaks the symmetries of the problem described in Fig. 2a.

To further quantify the transition between a planar shape into an out-of-plane equilibrium configuration, we measure the rod's maximum transverse displacement, $X_{\max }=\max (|X|)$, in the $(x, y)$ plane (defined in Fig. 2a and b) from the experimental images. In Fig. $3 \mathrm{a}$ and $\mathrm{b}$ we plot $X_{\max }$ as a function of the control parameter $\delta / L$, for the two values of natural curvatures considered above, $\kappa_{0}=16.5 \mathrm{~m}^{-1}$ and $\kappa_{0}=39.3 \mathrm{~m}^{-1}$. As $\delta / L$ is increased (decreasing the end-to end distance), the rod undergoes a symmetry-breaking pitchfork bifurcation for a critical displacement $\delta^{\text {crit }}$. Note that 

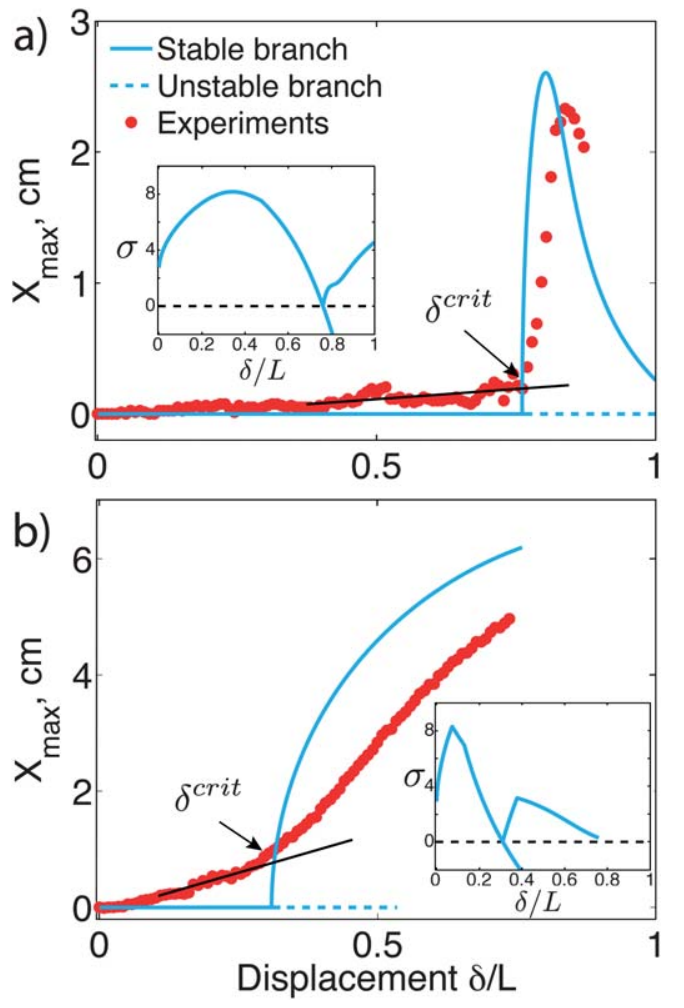

Fig. 3 Maximum transverse displacement, $X_{\max }$, as a function of $\delta / L$. (a) Case of a curved elastic rod with $\kappa_{0}=16.5 \mathrm{~m}^{-1}$. (b) Case of a curved elastic rod with $\kappa_{0}=$ $39.3 \mathrm{~m}^{-1}$. $X_{\max }$ is illustrated in Fig. 2a and b. Solid/dashed lines represent stable/ unstable semi-analytical branches obtained with simulations. Insets: evolution of the decay rate, $\sigma$, with control parameter $\delta / L$.

the pitchfork is qualitatively different for the two values of $\kappa_{0}$ considered, e.g. $X_{\max }$ is non-monotonic for low $\kappa_{0}$.

In parallel to the experiments, our numerical continuation method gives access to analytical branches of solutions of $X_{\max }$, as well as the associated decay rate $\sigma$ given in the inset of Fig. 3 (only the one branch, out of two, chosen by the experimental imperfections is plotted). The numerical displacement threshold, $\delta^{\text {crit }}$, is the value at which $\sigma$ becomes negative. We define the experimental threshold as the displacement region where there is a significant change of slope (larger than 5\%) in the evolution of $X_{\max }$, as a function of $\delta / L$ [see the straight black line in Fig. 3]. We observe a good overall quantitative agreement between numerical and experimental data, especially when comparing the instability threshold $\delta^{\text {crit }}$ obtained with both approaches. For $\kappa_{0}=16.5 \mathrm{~m}^{-1}$, the numerical and experimental results quantitatively agree and the pitchfork bifurcation leads to a clear instability threshold $\delta^{\text {crit }}$ located at a small end-to-end distance. For $\kappa_{0}=39.3 \mathrm{~m}^{-1}$, however, there is a significant difference between the simulated and experimental data (up to $36 \%$ ) although the threshold $\delta^{\text {crit }}$ is still correctly predicted at a larger end-to-end distance. This discrepancy may be attributed to the presence of residual internal twist or cross-sectional ellipticity that emerge in our fabrication process for high values of $\kappa_{0}$, or the sensitivity to imperfection of the smooth bifurcation process illustrated in the sequence of representative snapshots of Fig. 2b2-b4.
We proceed by further characterizing the influence of curvature in this writhing case I of imposed displacement. In particular, we aim to rationalize the pattern formation process by numerically extracting the internal forces stored in the rod. In Fig. 4a, we plot the phase diagram of our mechanical system where the phase boundary is given by the instability threshold $\delta^{\text {crit }} / L$ as a function of $\kappa_{0}$. The experimental data is in excellent agreement with the numerical predictions obtained from analyzing the decay rate $\sigma$. The phase diagram clearly delimits three regions of equilibrium configurations: the planar shape represented by the top view at point A in Fig. 4a, the anti-symmetric out-of-plane configuration illustrated at point B and the asymmetric state represented at C. Due to the complexity of behavior, e.g. involving self-contact, in the neighborhood of the phase boundary that delimits the two out-of-plane states (dotted line in Fig. 4a is a guide to the eye), we have decided to leave a detailed and systematic characterization of this region for a future study.

Another characteristic feature illustrated by Fig. $4 \mathrm{a}$ is the value of $\kappa_{0}$, denoted $\kappa_{0}^{\text {crit }}$, at which there is a sharp change in
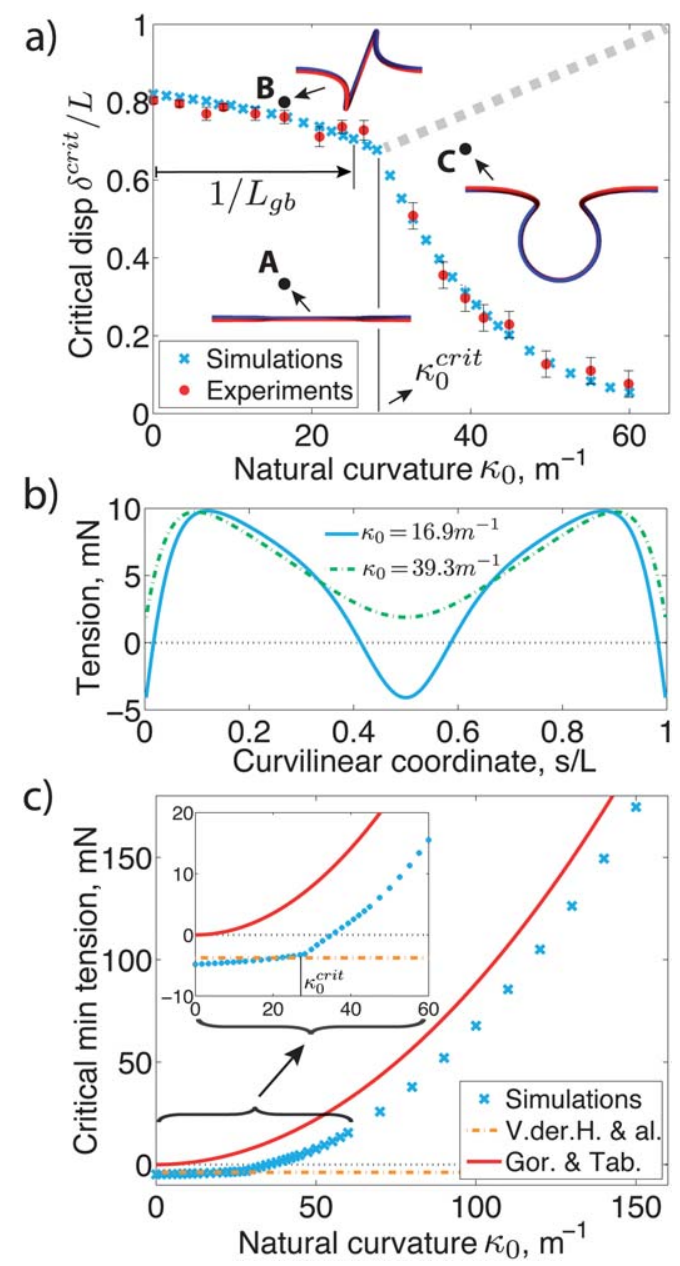

Fig. 4 Influence of natural curvature on case II. (a) Instability threshold $\delta^{\text {crit }} / L$ as a function of $\kappa_{0}$, for $\Phi=0^{\circ}$. A critical curvature $\kappa_{0}^{\text {crit }} \approx 1 / L_{\mathrm{gb}}$ clearly separates two different regimes (see text for details). (b) Evolution of tension along the rod just before $\delta^{\text {crit }} / L$ for values of $\kappa_{0}$ in the two different regimes. (c) Critical minimal tension in the rod at the instability threshold, as function of $\kappa_{0}$. Dot-dash line from ref. 26 and solid line from ref. 9. 
slope in the evolution of critical displacement $\delta^{\text {crit }} / L$, that delimits two distinct regimes. Interestingly, this critical curvature is comparable to the inverse of the elastogravity lengthscale $L_{\mathrm{gb}}$; the characteristic length above which the pull of gravity changes the natural shape of a naturally curved hanging rod. ${ }^{38-40}$ In our problem, $L_{\mathrm{gb}}=(E I / \rho g S)^{1 / 3}=24.7 \mathrm{~cm}$, where $E I=$ $E \pi R^{4} / 4$ is the bending modulus of the rod and $\rho S=\rho \pi R^{2}$ is its linear mass density. Below the critical curvature $\kappa_{0}^{\text {crit }} \approx 1 / L_{\mathrm{gb}}$, gravity is dominant and the rod behaves as a straight rod with a small imperfection. Above $\kappa_{0}^{\text {crit }} \approx 1 / L_{\mathrm{gb}}$, the effect of curvature becomes important and dominates the physics of the problem leading to the inversion of chirality observed in Fig. 2b2-b4.

In Fig. $4 \mathrm{~b}$, we represent the evolution of internal normal forces stored along the rod, immediately prior the onset of instability at $\delta^{\text {crit }} / L$, for two values of intrinsic curvatures $\kappa_{0}=$ $16.5 \mathrm{~m}^{-1}$ and $\kappa_{0}=39.3 \mathrm{~m}^{-1}$. The internal axial forces profile along the rods is qualitatively similar in both cases and minima of forces are located at the mid-point and extremities. However, for $\kappa_{0}=16.5 \mathrm{~m}^{-1}$, some parts of the rod are under compression whereas, for $\kappa_{0}=39.3 \mathrm{~m}^{-1}$, the whole rod is in tension at the onset of buckling. In Fig. 4c, we plot the evolution of critical minimum normal forces at onset of buckling as a function of $\kappa_{0}$ in the extended range $0<\kappa_{0}<150 \mathrm{~m}^{-1}$. Similarly to Fig. 4a, we can clearly identify the two regimes separated by $\kappa_{0}^{\text {crit }}$ in the evolution of critical axial forces highlighted by the zoom in the inset of Fig. 4c. This indicates that the mechanical response of the displacement experiment is dictated by the internal normal forces. In the first regime for $\kappa_{0} \lesssim 1 / L_{\mathrm{gb}}$, the elastic rod buckles out of the plane due to the compressive internal forces that eventually arise under large compressive displacements $\delta$. The critical compressive buckling load as a function of the imperfection $\kappa_{0}$ is fairly constant and can be reasonably compared to the critical buckling load, $F^{\text {crit }}=-6 \pi^{2} E I / L^{2}$ (dotted-dashed line in Fig. 4c), that has previously been found for a displacement experiment performed on a weightless nitinol rod with no intrinsic natural curvature. ${ }^{26,32}$ For $\kappa_{0} \gtrsim 1 / L_{\mathrm{gb}}$, where the effects of natural curvature become significant, the bifurcation process is markedly different. The critical internal axial force evolves quadratically in terms of $\kappa_{0}$, qualitatively following the solid line in Fig. $4 \mathrm{c}$ that represents the critical tension, $F^{\mathrm{crit}}=(1+\nu) E I \kappa_{0}{ }^{2}$. This analytical expression predicts the internal tension below which a twistless and weightless infinitely long elastic filament with intrinsic curvature $\kappa_{0}$ bifurcates onto a helix with inverted chirality. ${ }^{\mathbf{9}, 10}$ The convincing agreement between the theoretical and numerical results in Fig. 4c suggests that the buckling phenomenon for $\kappa_{0} \gtrsim 1 / L_{\mathrm{gb}}$ is due to a competition between elasticity, natural curvature and internal tension which, in our case, is provided by the rod's weight. We attribute the horizontal offset between the computed critical minimum tension with the analytical $F^{\text {crit }}$ to the delaying effect of gravity, that overcomes the destabilizing influence of natural curvature for $\kappa_{0} \leqq 1 / L_{\mathrm{gb}}$.

\section{Writhing case II - imposed rotation}

We now want to evaluate the robustness of the delay mechanism uncovered in the previous case I of imposed displacement by investigating the effect of $\kappa_{0}$ when we quasi-statically impose an end-to-end rotation $\Phi$ to the elastic rod, as illustrated in Fig. 1. This complementary test, performed for a fixed displacement $\delta / L=0.27$, begins at $\Phi=0^{\circ}$ and ends just before self-contact occurs. We study the mechanical response of the twisted rod in a similar way to case I, both experimentally and numerically.

In Fig. 2c and d, we present a series of representative experimental and numerical equilibrium configurations (top views), at the same values of control parameter $\Phi$. Again, we find excellent agreement between the two. For a straight rod with $\kappa_{0}=0 \mathrm{~m}^{-1}$, the rod initially exhibits a planar equilibrium shape that lies in the $(y, z)$ plane due to the effect of gravity as illustrated in Fig. 1 and 2c1. When the rotation angle $\Phi$ is increased, this initial planar shape evolves smoothly into an out-of-plane configuration, anti-symmetric with respect to the clamp's centerline, with an amplitude that grows due to an increasing internal twist [see Fig. $2 \mathrm{c} 2$ and c3]. At a critical value of the rotation angle, $\Phi^{\text {crit }} \approx 2040^{\circ}$, that is correctly predicted by our continuation method, the out-of-plane shape jumps into a plectoneme state, ${ }^{16,26}$ a highly localized structure consisting of a two-start right-handed helix with terminal loops, located in the middle of the rod. For a curved rod with $\kappa_{0}=44.8 \mathrm{~m}^{-1}$, the evolution of equilibrium configurations with the rotation angle $\Phi$ is qualitatively different as shown in Fig. 2 d. By introducing the constant natural curvature $\kappa_{0}$, the previously anti-symmetric out-of-plane solutions obtained for the case of straight rods become asymmetric with respect to the $(y, z)$ plane. For very small rotation angles $\Phi$ as illustrated in Fig. 2d1, the rod adopts a helical shape with opposite handedness as explained in the previous section for case I. Above a critical rotation angle, $\Phi=$ $395^{\circ},{ }^{36}$ our results confirm that the rod jumps into a one-twistper-wave helical mode due to the presence of natural curvature. ${ }^{28}$ In this configuration, the number of waves is equal to the number of full twists stored in the rod as shown in Fig. 2d1. For a critical rotation angle $\Phi^{\text {crit }} \approx 2960^{\circ}$, a plectoneme forms, superimposed onto the one-twist-per-wave equilibrium state. It is interesting to note that, for the naturally curved rod, the plectoneme is located at one extremity of the rod rather than at its center [see Fig. 2d4], as found above for the straight rod.

Similarly to case I, where we imposed displacement, but now for the end-to-end rotation experiment, in Fig. 5 we plot the rod's maximum transverse displacement, $X_{\max }$, as a function of the imposed rotation angle, $\Phi$. For the two values of $\kappa_{0}$ chosen, and across the full range of $\Phi$ explored, the experimental data is in excellent agreement with the numerical predictions (the accumulating discrepancies at high values of $\Phi$ in Fig. 5b may be interpreted by the absence of stretching in our numerical model). It is remarkable that the experimental instability threshold for the onset of a plectoneme, $\Phi^{\text {crit }}$, given also in Fig. $2 \mathrm{c} 4$ and $d 4$, is reproduced within $2 \%$ by our local numerical stability analysis. Unlike the relatively smooth response for $\kappa_{0}=$ $0 \mathrm{~m}^{-1}$ given in Fig. 5a, natural curvature eventually introduces a disconnected equilibrium branch as illustrated in Fig. 5b. Counterintuitively, we find that imparting a constant natural curvature to our rods (essentially adding a geometric imperfection to the stress-free configuration) results in considerably postponing (in this particular case, by approximately $43 \%$ ) the 

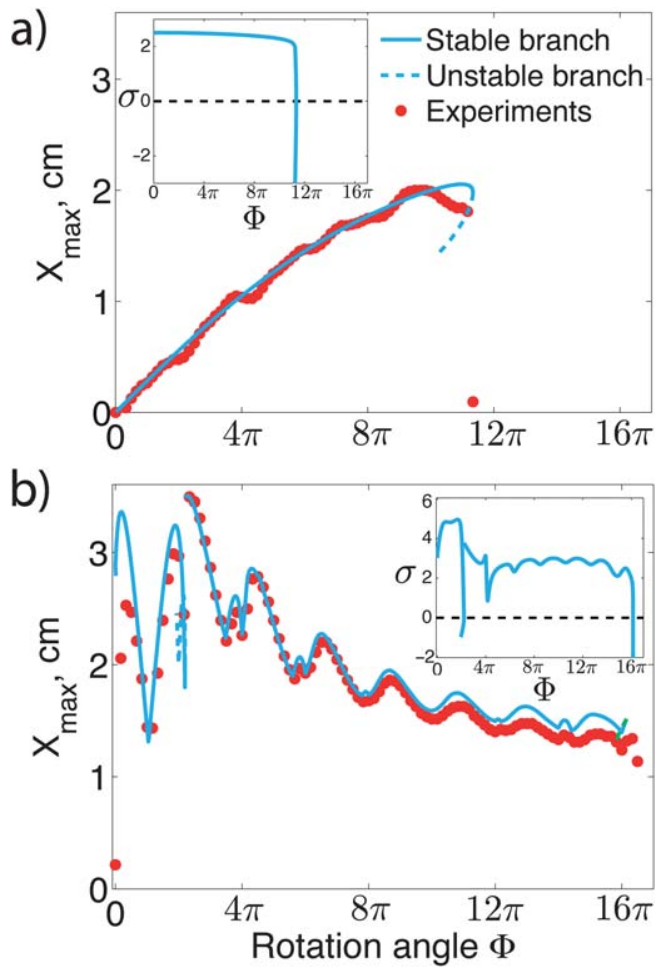

Fig. 5 Maximum transverse displacement, $X_{\max }=\max (\operatorname{abs}(X))$, as a function of $\Phi$. (a) Case of a straight elastic rod with $\kappa_{0}=0 \mathrm{~m}^{-1}$. (b) Case of a curved elastic rod with $\kappa_{0}=44.8 \mathrm{~m}^{-1}$. $X_{\max }$ is illustrated in Fig. $2 \mathrm{c}$ and d. Solid/dashed lines represent stable/unstable semi-analytical branches obtained from simulations. Insets: evolution of the decay rate, $\sigma$, with control parameter $\Phi$.

emergence of the plectoneme instability, which is often synonymous with failure in practical systems.

We now address how the natural curvature affects the mechanical response of the twisted rod. In Fig. 6, we plot the evolution of critical rotation angle $\Phi^{\text {crit }}$ as a function of $\kappa_{0}$, obtained from the experiments and simulations. Excellent agreement is found between the two, across the full range of explored $\kappa_{0}$. The constant natural curvature $\kappa_{0}$ strikingly postpones the emergence of the plectoneme. Moreover, this phenomenon is achieved through a nontrivial series of discrete steps, as $\Phi^{\text {crit }}$ is increased. And similarly to case I, the twisted heavy elastic rod is significantly affected by the imposed geometry only past a critical value of $\kappa_{0}$.

In Fig. 6A-E we present a sequence of equilibrium states, immediately after the onset of the plectoneme, for rods with increasing $\kappa_{0}$. The configurations A-E are each taken from the individual steps in the plot of Fig. 6. The deformed shapes associated with each step of $\Phi^{\text {crit }}$ correspond to states characterized by different number of waves that can be stored before the instability occurs and are directly related to the amount of twist required for the instability to be triggered. The signature of increasing $\kappa_{0}$ can also be seen on the position along the rod's arclengths, where the plectoneme forms. At low values of natural curvature (e.g. points A and B in Fig. 6), the plectoneme forms towards the middle of the $\operatorname{rod}(|s / L| \sim 0.5)$ as often mentioned in the literature, ${ }^{\mathbf{1 6 , 1 7}}$ whereas for higher curvature (e.g. points $\mathrm{C}-\mathrm{E}$ ) the plectoneme develops near the clamp $(|s / L| \sim 1)$.
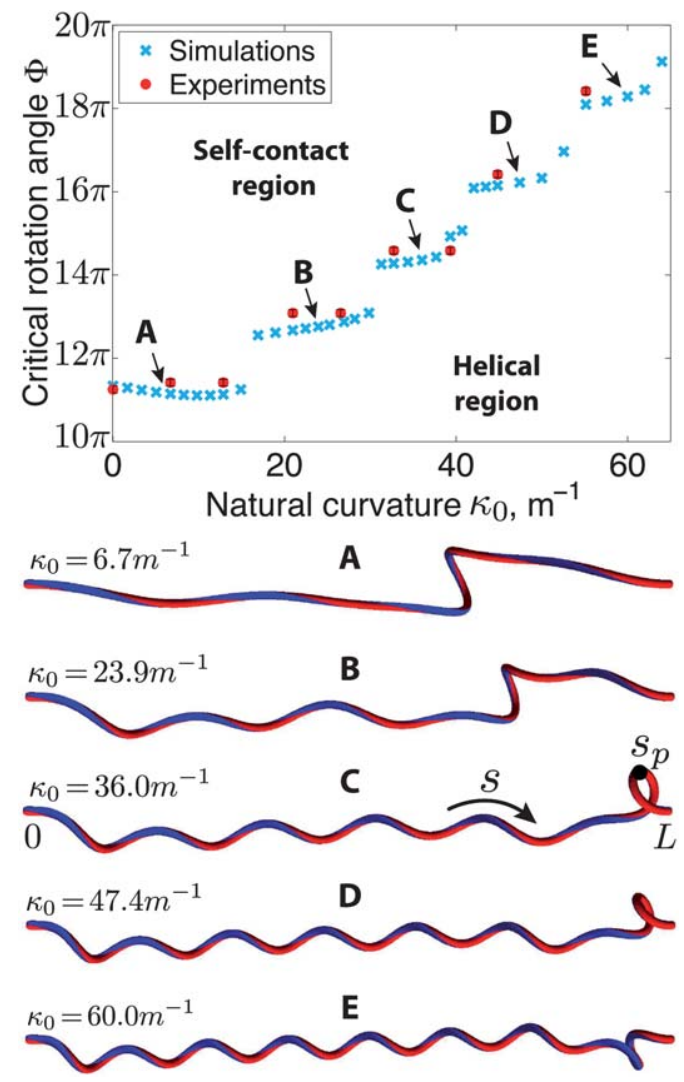

Fig. 6 Phase diagram of the rotation experiment. Instability threshold, $\Phi^{\text {crit }}$, as a function of natural curvature, $\kappa_{0}$, for $\delta / L=0.27$. The equilibrium configurations $A-E$, captured numerically right after onset of plectoneme are given for different values of $\kappa_{0}$ associated with each step of the phase diagram.

Making use of the simulations, we now connect the curvilinear coordinate at which the plectoneme forms, $s_{\mathrm{P}}$, to the internal tension and twist rate in the rod, both of which are plotted in Fig. 7a1 and a2, for different values of $\kappa_{0}$. For all the curvatures considered, the entire rod is in tension when the instability occurs, with a profile exhibiting a wavy shape that is closely related to the deformed configurations represented in Fig. 6A, B and D. In Fig. 7a2, the location of minimum tension at onset is associated to the location of minimum twist coincident with the position of plectoneme formation ${ }^{16}$ suggesting that internal axial forces have again a central role in the instability mechanism of the writhing case II. This finding is confirmed by the linear relation between the location of absolute maximum strain rates (both material curvatures and twist), $s_{\mathrm{P}}$, and minimum tension for the range of simulated natural curvatures $0<\kappa_{0}<60 \mathrm{~m}^{-1}$ illustrated in Fig. 7b. The inset in Fig. 7b also shows the complex progression of the location of the plectoneme with $\kappa_{0}$, confirming that plectonemes form closer to the edges as $\kappa_{0}$ increases. For small values of curvature, the localized pattern tends to emerge towards the middle of the rod, but there is a critical curvature, $\kappa_{0}^{\text {crit }}$, potentially related to the one uncovered in the case I of imposed displacement, above which plectonemes constantly appear at the extremities (apart for two data points where the rod reproducibly snaps into a central plectoneme, something which we have not yet been able to rationalize). 

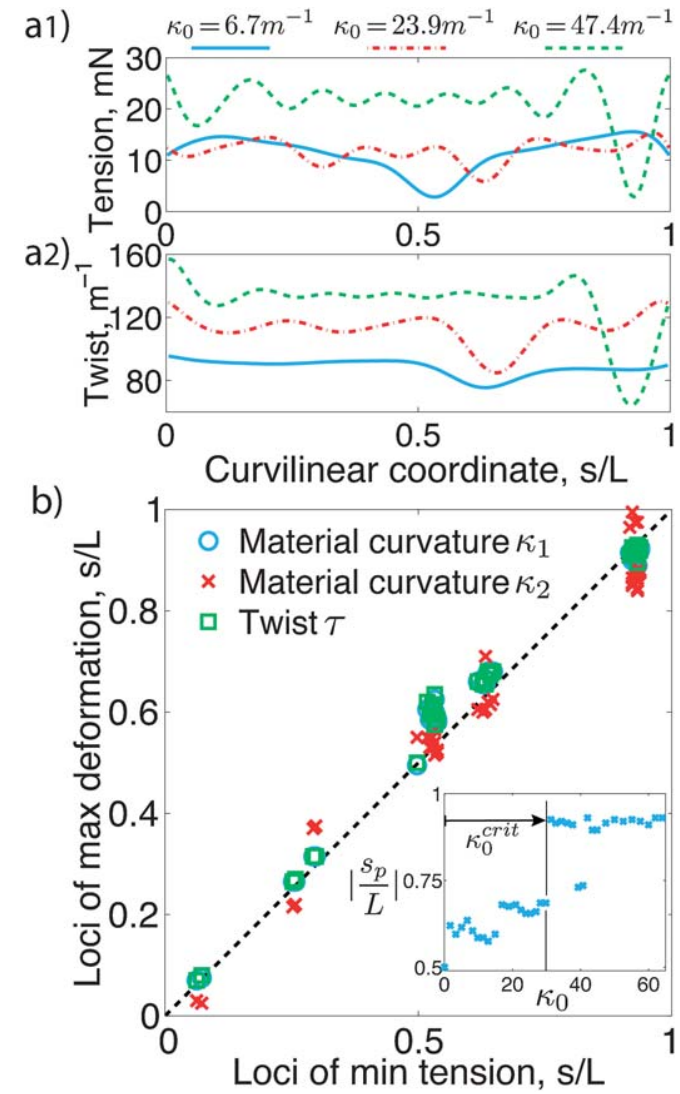

Fig. 7 Investigation on the location of plectoneme. (a) Profile of internal normal forces (a1) and twist rate (a2) along the rod at onset of plectoneme for different $\kappa_{0}$. (b) Location of plectoneme (identified as the maximum absolute flexural and torsional strain rate at instability) as a function of the minimal tension in the rod, right before the instability occurs. Inset: influence of $\kappa_{0}$ on the plectoneme location.

\section{Conclusions}

In summary, we have focused on the effect of natural curvature, $\kappa_{0}$, on the writhing of a heavy slender elastic rod, and presented results on the pattern formation of geometrically nonlinear configurations. Two complementary cases with particular imposed kinematics were considered for which we found a similar delaying mechanism where the influence of imperfection is discontinuous, with a length scale that we attribute to an elaborate interplay between natural curvature, elasticity and weight.

When imposing a compressive displacement, we found two regimes, separated by a critical curvature $\kappa_{0}^{\text {crit }}$ (seemingly related to the elastogravity length scale $L_{\mathrm{gb}}$ ) below which the rod behaves as a naturally straight rod with a geometric imperfection. Above $\kappa_{0}^{\text {crit }}$ the rod behaves as a naturally curved rod under tension. A similar delay in the influence of natural curvature due to weight was observed and explored in the more complex case of an imposed rotation. Surprisingly, we find that $\kappa_{0}$ postpones the emergence of plectonemes, in a stepwise manner, by adopting a one-twist-per-wave configuration, with increasing number of waves for higher curvatures. Furthermore, curvature qualitatively changes the plectoneme formation mechanism by shifting the plectoneme location from its center to the extremity of the rod.

Our findings could allow for the measurement, as an inverse problem, of the natural curvature of a heavy elastic rod. Moreover, our work points to a novel way to control localized instabilities in slender wires, cables and tubes by tuning their intrinsic curvature. Whereas we have focused on the role of gravity, the prominence of geometry in this class of problems suggests that the framework we propose and the mechanism we identify should be relevant to other systems, over a wide range of length scales, where conservative body forces and natural curvature coexist in the mechanical response of soft filamentary structures.

\section{Acknowledgements}

We thank Basile Audoly for useful discussions. We acknowledge funding from the National Science Foundation (CMMI1129894), Schlumberger-Doll Research, MIT's Undergraduate Research Opportunity Program (M.M.) and the Battelle-MIT postdoctoral fellowship program (A.L.).

\section{References}

1 S. Neukirch, Phys. Rev. Lett., 2004, 93, 198107.

2 D. Garrivier and B. Fourcade, Europhys. Lett., 2000, 49, 390395.

3 J. Marko and S. Neukirch, Phys. Rev. E: Stat., Nonlinear, Soft Matter Phys., 2012, 85, 011908.

4 R. S. Manning, K. A. Rogers and J. H. Maddocks, Proc. R. Soc. London, Ser. A, 1998, 454, 3047-3074.

5 P. Furrer, R. Manning and J. Maddocks, Biophys. J., 2000, 79, 116-136.

6 A. Balaeff, L. Mahadevan and K. Schulten, Phys. Rev. E: Stat., Nonlinear, Soft Matter Phys., 2006, 73, 031919.

7 R. Goldstein, A. Goriely, G. Huber and C. Wolgemuth, Phys. Rev. Lett., 2000, 84, 1631-1634.

8 C. Darwin, The movements and habits of climbing plants, J. Murray, 1888.

9 A. Goriely and M. Tabor, Phys. Rev. Lett., 1998, 80, 1564-1567.

10 T. McMillen and A. Goriely, J. Nonlinear Sci., 2002, 12, 241281.

11 T. Yabuta, Bull. JSME, 1984, 27, 1821-1828.

12 S. Goyal, N. Perkins and C. Lee, Int. J. Nonlinear Mech., 2008, 43, 65-73.

13 A. Greenhill, Proc. - Inst. Mech. Eng., 1883, 34, 182-225.

14 A. Love, A treatise on the mathematical theory of elasticity, University Press, 1920.

15 J. Coyne, IEEE J. Oceanic Eng., 1990, 15, 72-83.

16 J. Thompson and A. Champneys, Proc. R. Soc. London, Ser. A, 1996, 452, 117-138.

17 A. Goriely and M. Tabor, Proc. R. Soc. London, Ser. A, 1998, 454, 3183-3202.

18 A. Goriely and M. Tabor, Proc. R. Soc. London, Ser. A, 1997, 453, 2583-2601.

19 M. Davies and F. Moon, Chaos, 1993, 3, 93. 
20 A. Champneys and J. Thompson, Proc. R. Soc. London, Ser. A, 1996, 452, 2467-2491.

21 A. Goriely and M. Tabor, Physica D, 1997, 105, 20-44.

22 A. Goriely and M. Tabor, Physica D, 1997, 105, 45-61.

23 S. Neukirch, G. Van Der Heijden and J. Thompson, J. Mech. Phys. Solids, 2002, 50, 1175-1191.

24 Y. Miyazaki and K. Kondo, Int. J. Solids Struct., 1997, 34, 3619-3636.

25 G. Van der Heijden and J. Thompson, Nonlinear Dyn., 2000, 21, 71-99.

26 G. Van der Heijden, S. Neukirch, V. Goss and J. Thompson, Int. J. Mech. Sci., 2003, 45, 161-196.

27 E. Michael and S. Neukirch, Int. J. Bifurcat. Chaos, 2004, 14, 1223-1239.

28 A. Champneys, G. Van der Heijden and J. Thompson, Philos. Trans. R. Soc., A, 1997, 355, 2151-2174.

29 B. Coleman and D. Swigon, J. Elasticity, 2000, 60, 173-221.

$30 \mathrm{~F}$. Liu and N. Center, Kink formation and rotational response of single and multistrand electromechanical cables, Defense Technical Information Center, 1975.
31 Y. Yang and S. Kuo, J. Struct. Eng., 1987, 113, 1185-1202. 32 V. Goss, G. van der Heijden, J. Thompson and S. Neukirch, Exp. Mech., 2005, 45, 101-111.

33 J. Thwaites, J. Text. Inst., 1970, 61, 116-138.

34 W. K. Silk, Environ. Exp. Bot., 1989, 29, 95-109.

35 K. Newman and D. Newburn, SPE Annual Technical Conference and Exhibition, 1991.

36 A. Lazarus, J. Miller and P. Reis, J. Mech. Phys. Solids, 2013, 61, 1712-1736.

37 S. Karkar, R. Arquier, A. Lazarus, O. Thomas, C. Vergez and B. Cochelin, Manlab: An interactive path-following and bifurcation analysis software, http://manlab.lma.cnrs-mrs.fr/, 2010.

38 A. Föppl, Vorlesungen über technische Mechanik: Graphische Statik. 7, BG Teubner, 1912, vol. 2.

39 B. Audoly and Y. Pomeau, Elasticity and geometry: From hair curls to the nonlinear response of shells, Oxford Press, 2010.

40 P. Reis, J. Hure, S. Jung, J. Bush and C. Clanet, Soft Matter, 2010, 6, 5705-5708. 